\title{
An openEHR Approach to Detailed Clinical Model Development: Tobacco Smoking Summary Archetype as a Case Study
}

\author{
Ping-Cheng Wei ${ }^{1}$ Koray Atalag ${ }^{1}$ Karen Day ${ }^{1}$ \\ ${ }^{1}$ School of Population Health, The University of Auckland, \\ New Zealand, Auckland, New Zealand \\ Address for correspondence Karen Day, PhD, The University of \\ Auckland, Private Bag 92019, Auckland 1142, New Zealand \\ (e-mail: k.day@auckland.ac.nz).
}

Appl Clin Inform 2019;10:219-228.

\begin{abstract}
Keywords

- electronic health records

- knowledge management

- data creation

- data modeling

- archetypes

Background Data modeling for electronic health records (EHRs) is complex, requiring technological and cognitive sophistication. The openEHR approach leverages the tacit knowledge of domain experts made explicit in a model development process aiming at interoperability and data reuse.

Objective The purpose of our research was to explore the process that enabled the aggregation of the tacit knowledge of domain experts in an explicit form using the Clinical Knowledge Manager (CKM) platform and associated assets. The Tobacco Smoking Summary archetype is used to illustrate this.

Methods Three methods were used to triangulate findings: (1) observation of CKM discussions by crowdsourced domain experts in two reviews, (2) observation of editor discussions and decision-making, and (3) interviews with eight domain experts. CKM discussions were analyzed for content and editor discussions for decision-making, and interviews were thematically analyzed to explore in depth the explication of tacit knowledge.

Results The Detailed Clinical Model (DCM) process consists of a set of reviews by domain experts, with each review followed by editorial discussions and decisionmaking until an agreement is reached among reviewers and editors that the DCM is publishable. Interviews revealed three themes: (1) data interoperability and reusability, (2) accurate capture of patient data, and (3) challenges of sharing tacit knowledge.

Discussion The openEHR approach to developing an open standard revealed a complex set of conditions for a successful interoperable archetype, such as leadership, maximal dataset, crowdsourced domain expertise and tacit knowledge made explicit, editorial vision, and model-driven software. Aggregated tacit knowledge that is explicated into a DCM enables the ability to collect accurate data and plan for the future.

Conclusion The process based on the CKM platform enables domain experts and stakeholders to be heard and to contribute to mutually designed standards that align local protocols and agendas to international interoperability requirements.
\end{abstract}

received

August 24, 2018

accepted after revision

January 31, 2019 (c) 2019 Georg Thieme Verlag KG Stuttgart · New York
DOI https://doi.org/

10.1055/s-0039-1681074. ISSN 1869-0327. 


\section{Background and Significance}

The prevalence of tobacco smoking presents a grand challenge for health care systems worldwide. The characteristics of tobacco smoking incorporate multiple interacting factors that stem from within and beyond the individual self. To add to the challenge, disagreement among stakeholders and limited access to resources create contention and obscure the general sense of direction as to how the rising burden of disease should be addressed. ${ }^{1}$ The development of health information technology (HIT) solutions can be just as difficult. Through exploring the international review and subsequent publication of the Tobacco Smoking Summary archetype, we aim to assess the implications of the openEHR approach to developing a consensus-based comprehensive dataset that can meet various demands within the tobacco smoking landscape, highlighting innovative qualities in modeling complex health care concepts.

The emergence of electronic health records (EHRs) has significantly improved the way in which patient health data are stored, retrieved, and used. $^{2}$ EHRs are real-time longitudinal records that provide a broad overview of a patients' health. The records are accessible by both patients and authorized health care professionals involved in care delivery, thereby improving the coordination, collaboration, and integration of health care services. While the primary aim of EHRs is to support care delivery by improving clinical documentation and enabling automated decision support, secondary use of EHR data also provides an excellent opportunity to support population health, health research, and policy making. Aggregation of patient data from disparate sources enables a better understanding of a population's health characteristics relevant to a specific health condition. ${ }^{3}$ However, there are serious impediments for being able to aggregate and reuse health care data from various sources in terms of data content and format compatibility, or, in other words, interoperability. ${ }^{4}$

The openEHR Foundation is an open standards organization that strives to achieve universal interoperability and reusability of all electronic patient health data. ${ }^{5}$ The International Telecommunications Union (ITU) defines open standards as "standards made available to the general public and are developed (or approved) and maintained via a collaborative and consensus driven process." As such, the openEHR Foundation is an open standards organization and exhibits ITU characteristics such as collaboration, due process, intellectual property rights (which leverage Creative Commons), balanced contributions from crowdsourced experts, transparency, high-quality decision-making, ongoing support, and publicly available archetypes. ${ }^{6}$ Detailed Clinical Models (DCMs), called "archetypes" in openEHR, are computable structured representations of health care concepts and complex clinical expressions that are documented in EHRs. ${ }^{7}$ Health information is modeled through a combination of expert knowledge, data specifications and terminologies, and various business and technical requirements. DCMs allow for a consistent collection of complex health care data that can then be shared and aggregated to drive automation in care delivery and also facilitate secondary use. ${ }^{8}$
The openEHR supports model-driven development (MDD) and employs a two-level modeling method for DCM development. ${ }^{9}$ The first level of modeling comprises a stable reference model (RM) upon which software is built. The $\mathrm{RM}$ is the base information model for all openEHR systems and defines the invariant structure, data types, and semantics of health care data. The second level comprises archetypes and templates, which use RM as building blocks to define reusable clinical content such as blood pressure measurements or laboratory results. ${ }^{10-12}$ The openEHR template is a logical representation of case-specific datasets that bring together and further constrain archetypes such as a discharge summary or pathology report. ${ }^{10}$

In MDD methodology, the term model refers to visual representations of simplified abstractions, each one specifying a different view of a complex domain, and software code can be automatically generated from these models in software development. MDD allows for separation of technical and business viewpoints and tasks. This enables software developers to create information systems without the need for understanding domain concepts and detailed requirements, simultaneously allowing for domain experts to specify information requirements and business logic using technology-agnostic domain models that use visual modeling tools. ${ }^{12-14}$ In openEHR, archetypes and templates can be authored by domain experts; therefore, DCM development can progress without indepth understanding of software development. Consequently, more time and effort can be invested in developing the clinical content of the archetype. ${ }^{15,16}$

The openEHR Clinical Knowledge Manager (CKM) is an online collaborative e-health knowledge asset-management application and repository of clinical knowledge resources such as archetypes, templates, and clinical terminology subsets. ${ }^{17}$ It allows domain experts with different backgrounds and levels of experience to collaborate, cooperate, and contribute to the development, review, and maintenance of DCMs. ${ }^{18,19}$ Participants include openEHR editors (who set the vision for a DCM and manage the process) and domain experts from within the field of interest, in this case tobacco smoking. The editors recruit domain experts through personal contacts from their network and an open invitation on the openEHR Web site. The domain experts' contributions to a DCM constitute a form of crowdsourcing. ${ }^{8,9}$ The aggregation of their tacit knowledge provides fresh perspectives and ensures that a DCM is developed in a manner that enables it to meet the ever-shifting health demands of populations. ${ }^{4,10}$

Tacit knowledge is the knowledge that people carry in their heads; it is not documented in any way and is only accessible by the person who has it. ${ }^{20,21}$ This unarticulated knowledge is associated with movement skills, intuition, implicit general rules, the senses, and physical experiences. $^{22}$ Some tacit knowledge is unspecifiable (cannot be made explicit), and other knowledge is implicit because it can be made explicit, for example, documented in models such as the DCM for tobacco smoking. ${ }^{21}$ The CKM approach aims to explicate as much tacit knowledge as possible for the formation of a maximal dataset, which forms the basis of a DCM. 
DCM development starts with identifying existing relevant artifacts, which can be reused completely or adapted for a particular need, which is key for interoperability. A new DCM development process consists of an initial model design followed by iterative reviews (roughly 2 weeks of duration each). Domain experts discuss aspects of a model until convergence occurs. After each iteration, the editors make decisions about gaps, overlaps, and divergence in the expert discussion. Reviews are repeated until the DCM under development is sufficiently complete for publication on the openEHR Web site. ${ }^{23}$ These aspects of the openEHR CKM create an environment that simplifies typically error-prone manual operations and complex workflows, and ensure the efficient and effective online governance of e-health knowledge assets. ${ }^{4,18}$

Within the context of health information models and interoperability, openEHR and Health Level Seven's (HL7) Fast Healthcare Interoperability Resources (FHIR) are well-known standards. There is a debate about whether they are competing or complementary. HL7's FHIR is an emerging standard, which also defines structured and reusable information models (called "resources") to support simpler and a limited number of use cases for the purpose of health information exchange. ${ }^{24} \mathrm{~A}$ few of these resources are considered DCM, such as Condition and AllergyIntolerance, whereas most others resemble openEHR RM building blocks that are domain invariant. More complex DCMs can be created by profiling, where resources can be aggregated and further constrained, similar to openEHR templates..$^{25}$ Leveraging modern Web standards and technologies, FHIR is used to create simple, lightweight application programming interfaces for quickly building data-driven applications and also supports terminology bindings. For the purpose of our research, we chose openEHR, which is more comprehensive.

\section{Objectives}

The purpose of our research was to explore the process that enabled the aggregation of the tacit knowledge of domain experts in an explicit form using the CKM platform and associated assets. We describe the DCM development pro- cess and contributions of reviewers to the Tobacco Smoking Summary archetype to illustrate this. We examine the expectations of domain experts who contributed to the model development, the openEHR features, and the success factors and future proofing of the published DCM.

\section{Methods}

Two perspectives of the openEHR approach of particular interest to the study were the review mechanics (DCM development process) and human cognition (domain expertise). The Tobacco Smoking Summary DCM was already under development at the time of the research. We approached a CKM editor for permission to observe the review activities and further development of the archetype.

One researcher (K. A.) was an existing registered CKM reviewer. The second researcher (P. W.) registered as a CKM reviewer to observe the two review periods ( - Fig。 1). - Fig. 1 depicts the review timeline and CKM activities (row 1 ), and the research methods matched to each CKM activity (rows 2 and 3 ).

Each review consisted of (1) the CKM editors' presentation of the latest version of the DCM, (2) online reviewer feedback on the DCM through the CKM platform, and (3) a discussion by the editors of the reviewers' contributions and decisions about further adjustments to the model. The online review comments were printed and treated as a document for summative content analysis (rather than conventional or directed content analysis). ${ }^{26}$ The CKM reviewers were expected to achieve a degree of consensus on aspects of the DCM raised by their colleagues and themselves, that is, the purpose of each review round. We counted the number of comments per review and identified instances of consensus and divergence from the content, comparing the first review round with the second round. We coded the content of the discussions in the CKM and clustered the codes. Since we were interested in how participants converged on consensus regarding the maturation of the DCM rather than the discourse itself that informed their consensus building, we did not use discourse analysis. ${ }^{27}$

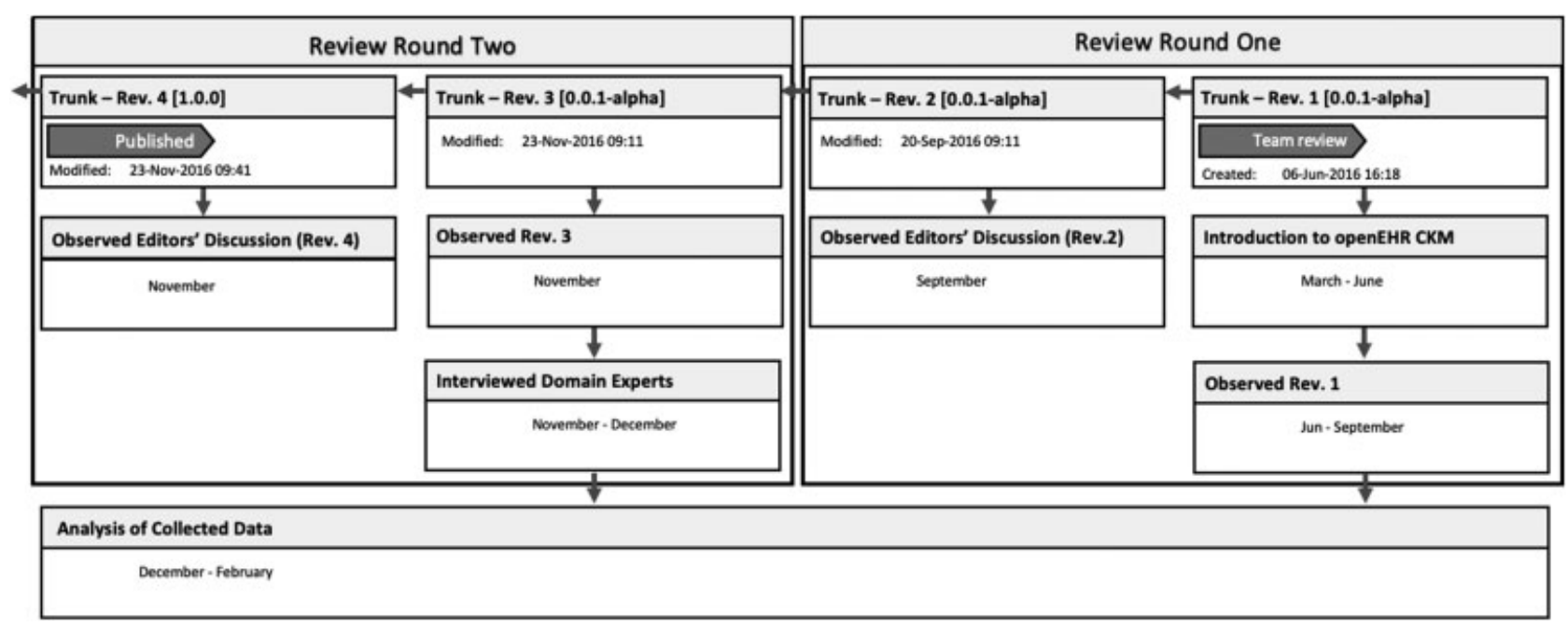

Fig. 1 Timeline for Clinical Knowledge Manager activities and matched data gathering activities. 
The editors' discussions were held through videoconference and were observed by P. W., who made digital voice recordings and written field notes. These were transcribed and thematically analyzed. ${ }^{28}$ This involved reading and rereading the transcriptions to become immersed in the data followed by coding of topics, which, in turn, were clustered into emergent themes. ${ }^{28,29}$ Again, since we expected convergence on consensus with reasons supplied, we chose to use thematic analysis rather than discourse analysis. The same analysis approach was used for analyzing the interviews.

After the reviews concluded and the DCM was published, reviewers were invited to participate in semistructured interviews about their experience of the review and DCM building, and expertise on the subject matter of the Tobacco Smoking Summary DCM. Interview questions were derived from the literature and observation of two review periods.

The interviews started with questions about reviewers' profession and interest in tobacco use data and about their exposure of or experience of DCMs and standards such as openEHR, HL7, ISO/EN [International Organization for Standardization, English version], and others. They were then asked about tobacco use data that they work with-how is it collected, how it is designed with multiprofessional input, its maintenance (by whom and how often), how well it satisfies the needs of a wide range of users in health care, whether it provides a complete and accurate representation of behaviors surrounding tobacco use, and improvements to enable users to understand tobacco use better. Interviewees were then asked about their prior involvement in the developmental process of DCMs, the importance of end-user participation in DCM development, and whether multiprofessional collaboration is likely to meet end-user needs. They were asked what they felt was the most important aspect of the developmental process of DCMs, their experience of participating in an online community involved in development and governance of DCMs, and what concerns and expectations they might discuss with such a group. Finally, they were asked for their opinion on achieving the right balance between capturing local- or project-specific information requirements and satisfying the needs of global or national use cases-what is the sweet spot between local interoperability and global interoperability? Responses were probed with "why/why not" questions to gain deeper insights.

The interviews were digitally recorded and transcribed. These data were analyzed using a thematic analysis approach as described for the analysis of the editorial discussions. ${ }^{28,29}$ One author, P. W., conducted the interviews, and the other two authors checked the data analysis done by P. W. Issues arising from the data analysis were discussed by all three authors to explore and resolve difficulties and disparities.

\section{Results}

\section{Review Process and Content Analysis}

Observation of the CKM contributions revealed that it took two review rounds to develop the Tobacco Smoking Summary DCM. Each review round consisted of an online dis-
Table 1 Summary of reviewer and editor comments

\begin{tabular}{|l|l|l|}
\hline & $\begin{array}{l}\text { Review } \\
\text { round 1 }\end{array}$ & $\begin{array}{l}\text { Review } \\
\text { round 2 }\end{array}$ \\
\hline Number of reviewers & 10 & 15 \\
\hline Total reviewer comments & 52 & 49 \\
\hline $\begin{array}{l}\text { Comments regarding accurate } \\
\text { data capture }\end{array}$ & 26 & 7 \\
\hline $\begin{array}{l}\text { Comments regarding data } \\
\text { interoperability and reusability }\end{array}$ & 5 & 2 \\
\hline \begin{tabular}{l} 
Comments regarding both aspects \\
\hline $\begin{array}{l}\text { Additional comments (e.g., "yes," } \\
\text { "agree," "same as above" in } \\
\text { response to editorial feedback) }\end{array}$
\end{tabular} & 7 & 91 \\
\hline Total editor comments & 50 & 19 \\
\hline $\begin{array}{l}\text { Comments agreeing to } \\
\text { revision/change }\end{array}$ & 16 & 13 \\
\hline $\begin{array}{l}\text { Comments justifying no } \\
\text { review/change }\end{array}$ & 32 & 5 \\
\hline Additional comments & 2 & 1 \\
\hline
\end{tabular}

cussion for up to 2 weeks of duration followed by a videoconference discussion by editors. One of the six CKM editors uploaded a relatively mature DCM for Tobacco Smoking Summary onto the CKM platform for the first observed review. Ten domain experts contributed 52 comments to the first review we observed (review round 1) and 15 contributed 49 comments to the second review (-Table 1). Six editors contributed to the editorial discussion for each review. The editors examined the reviewers' contributions. They focused on the development of a universal maximal dataset that accommodates the requirements of various sectors across the health care system.

After each review, the editors met and discussed the contributions and decided which content would be included and whether the model was ready for release and use or required another review. The editors then upload the updated version of the Tobacco Smoking Summary archetype onto the openEHR CKM platform and left comments for the reviewers regarding their decisions. There were 50 editor comments after the first observed review and 19 after the second, indicating that the need for further discussion was coming to an end. During the editorial discussions, a bias appeared to emerge. The tacit knowledge of reviewers appeared to assume minimal datasets as the DCM foundation, and this had to be shifted to the editorial vision of maximal datasets. The editors assessed the reviewer contributions and realigned them to the requirement for a maximal dataset.

After a total of two review rounds, the editors decided that the DCM was ready for publication and use (with a view to future review should the need arise). This becomes clear in -Table 2, where the editors' decisions shifted from revision to acceptance.

The crowdsourcing of domain experts provided the editors with tacit knowledge that expanded their views and informed their decisions as to how the DCM was to be 
Table 2 Summary of editorial decisions

\begin{tabular}{|l|l|l|}
\hline & $\begin{array}{l}\text { Review } \\
\text { round 1 }\end{array}$ & $\begin{array}{l}\text { Review } \\
\text { round 2 }\end{array}$ \\
\hline Acceptance of archetype & 3 & 10 \\
\hline $\begin{array}{l}\text { Minor revision of } \\
\text { archetype needed }\end{array}$ & 4 & 5 \\
\hline $\begin{array}{l}\text { Major revision of } \\
\text { archetype needed }\end{array}$ & 3 & 0 \\
\hline Rejection of archetype & 0 & 0 \\
\hline
\end{tabular}

developed. As the reviews and editorial discussions progressed, signs of maturity in the model appeared in the form of diminishing comments from both reviewers and editors ( - Table 1 ) and a shift to acceptance and publication $(-$ Table 2 and - Fig. 1 ). The summative content analysis and observation revealed the importance of leadership and management by the editors, and the explication of reviewers' tacit knowledge into comments that resulted in refinement of the Tobacco Smoking Summary archetype.

The Tobacco Smoking Summary archetype is complex and has 51 data elements. -Fig. 2 is a screengrab of a high-level diagram of the archetype.

Due to the DCM's complexity and details, it is difficult to present the full details of changes after each revision in a concise way within this manuscript. - Table 3 depicts a summary of the modifications that were made to the model. Details of changes to the archetype are available on the openEHR website. ${ }^{30}$ The changes diminished from one
Table 3 Summary of nature of modifications made to the DCM

\begin{tabular}{|l|l|l|l|}
\hline $\begin{array}{l}\text { Review } \\
\text { comparison }\end{array}$ & Initial vs. R1 & R1 vs. R2 & Publication \\
\hline $\begin{array}{l}\text { Total } \\
\text { modifications }\end{array}$ & 21 & 14 & 5 \\
\hline $\begin{array}{l}\text { Modifications } \\
\text { (description) }\end{array}$ & 7 & 7 & 0 \\
\hline $\begin{array}{l}\text { Modifications } \\
\text { (definition) }\end{array}$ & 2 & 2 & 0 \\
\hline $\begin{array}{l}\text { Modifications } \\
\text { (ontology) }\end{array}$ & 12 & 5 & 5 \\
\hline $\begin{array}{l}\text { Overall } \\
\text { compatibility }\end{array}$ & Minor & Minor & Major $^{\mathrm{a}}$ \\
\hline $\begin{array}{l}\text { Contributor } \\
\text { comparison }\end{array}$ & +8 & +7 & 0 \\
\hline
\end{tabular}

Abbreviation: DCM, Detailed Clinical Model.

apublication itself is considered a major revision.

review to the next as consensus occurred, and the final change was publication.

\section{Themes Emerging from the Interviews}

All the reviewers and editors were invited to participate in the interviews as we were interested in knowing how their tacit knowledge and differing perspectives influenced their contribution to the archetype. Participants came from Australia, Brazil, England, New Zealand, Norway, and Sweden. Data saturation (i.e., no new content) ${ }^{31}$ was achieved in the eighth interview, at which point we discontinued interviewing. The

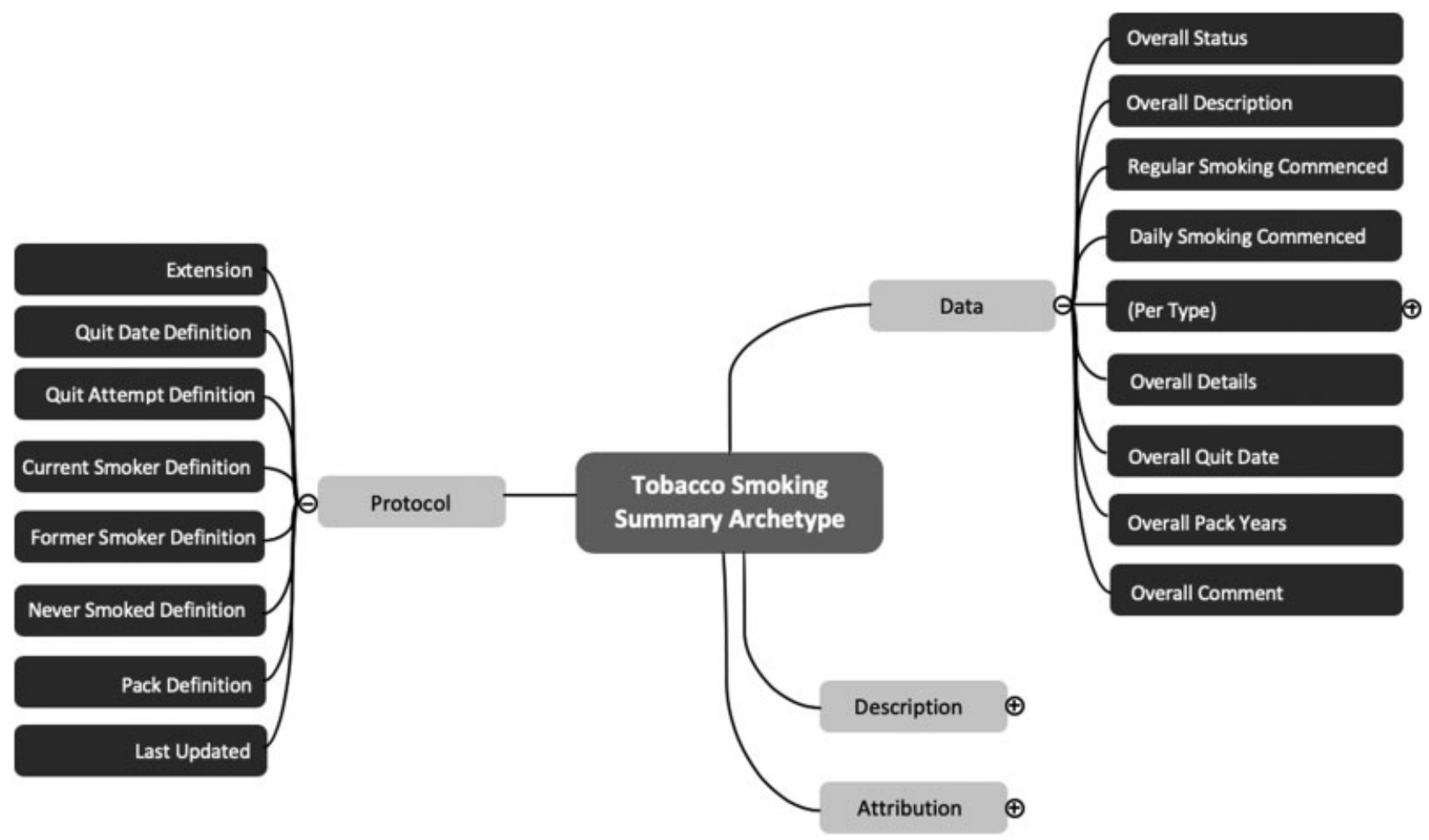

Fig. 2 High-level screengrab of Tobacco Smoking Summary archetype in a mind map format. 
following three themes emerged: (1) data interoperability and reusability, (2) accurate capture of patient smoking data, and (3) challenges of sharing tacit knowledge.

Data interoperability is essential for data reuse: successful data interoperability was considered by interviewees as a key factor that determines the success of health information systems, which, in turn, allows for data reusability. They attributed the significance of data interoperability to data communication, which subsequently influences cooperation and collaboration, and ultimately the adoptability of an overall system. The complexity of achieving data interoperability is closely attributed to the diverse demographics and smoking culture around the world. The degree to which interoperability can be achieved, however, is largely dependent on time, location, and demography, as illustrated by this interviewee's comment,

"There is no ideal sweet spot between local and international interoperability, it varies enormously depending on time and place."

Accurate data capture is essential for quality clinical care: the degree of detail in the data captured within a system determines its accuracy in representing the population it serves. As a targeted population (and use-case) increased, the number of data elements that are captured also increased. For example, the content reviews revealed that tobacco users in Nordic countries commonly use snus (tobacco is placed under the upper lip instead of being smoked). Should snus be included, as it is only used in Nordic countries? Leaving it out of the archetype would result in inaccurate data representation of that population's tobacco use. A challenging aspect of the archetype development is deciding the depth of detail in data to be captured. This, in turn, affects how the captured data will accurately represent the smoking characteristics of patients and enable health care providers to make informed decisions regarding their patients' care. The following quote from an interview illustrates the challenge:

"Different countries have different risks, exposures, and nomenclatures, so it is hard to develop a scale that would make sense in all the different countries."

Data capturing should therefore be easy and lend itself toward accuracy in the clinical setting with diverse needs for and responses to data. Maximal datasets therefore offer flexibility and sensitivity in complex environments.

Sharing tacit knowledge can be challenging: the features of openEHR CKM enable the conversion of domain experts' tacit knowledge into explicit knowledge as they develop an archetype for multiple uses. In the content reviews, the reviewers contribute to the archetype development from the perspective that relates to the demography they operate in and, by doing so, present the (minimal) datasets they are interested in. The editors are then in a position to assess the different minimal datasets (reviewer contributions) and, in conjunction with their dataset expectations, identify data elements that align with a maximal dataset. Knowledge sharing is complex and ambiguous. Interviewees commented on CKM as a platform that enabled a community of diverse domain experts to cooperate, collaborate, and contribute to the online knowledge resource (in this case, the Tobacco Smoking Summary archetype). The resulting dataset is the one that can be successfully adopted by various sectors of the health care system and can create an environment where patient data are interoperable and reusable.

Following the science and practice of health informatics, the domain experts recommended that one should assess whether similar scenarios have been captured before and use existing knowledge and experience (i.e., explicit knowledge) as a foundation to learn and build on. With this approach, any of the preliminary steps leading up to the development of a health information system can be bypassed so that more time and effort can be invested in the quality of its content. To the best of our knowledge, there is currently no system developed with this feature and nor are there any attempts to create one. The potential for developers to search for specific use-cases along with corresponding solutions highlights a promising concept that could benefit the future of health information systems development.

\section{Discussion}

\section{The openEHR Approach to Detailed Clinical Model Modeling}

In this study, we have described the DCM development process, the contribution of domain experts, and the vision and leadership provided by the CKM editors by observing two review rounds of the developing Tobacco Smoking Summary archetype. The interviews with contributing domain experts revealed insights about the use of DCMs for achieving interoperability and data reusability, the importance of accurate data capture for quality clinical care, and challenges associated with tacit knowledge sharing.

The openEHR approach to archetype development reveals a complex process that incorporates several interdependent aspects that determine the success of an archetype to accurately capture patient data and achieve data interoperability and reusability. - Fig. 3 depicts these aspects. On the left side of the diagram, tacit knowledge (knowledge that is not yet documented) is elicited from volunteer domain experts who form a community on the CKM platform. A DCM is developed and matures using the principle of maximal datasets, which, in turn, contributes to accurate data capture, resulting in interoperability. On the right side of the diagram, editorial leadership contributes to the explication of tacit knowledge in the form of the DCM (conversion of knowledge from tacit to explicit ${ }^{20}$ ), which, in turn, supports model-driven software development while influencing maximal dataset development, accurate data collection, and interoperability. Key success factors relating to the openEHR approach are the (1) availability of the openEHR community of domain experts, (2) MDD methodology, (3) reviewers' tacit knowledge made explicit in the reviews, and (4) editors' vision and synthesis of experts' contributions. These aspects align with the sociotechnical characteristics of archetype development, linking human cognition (tacit knowledge of reviewers and editors) to the mechanics of the archetype development process. ${ }^{2,12}$ 


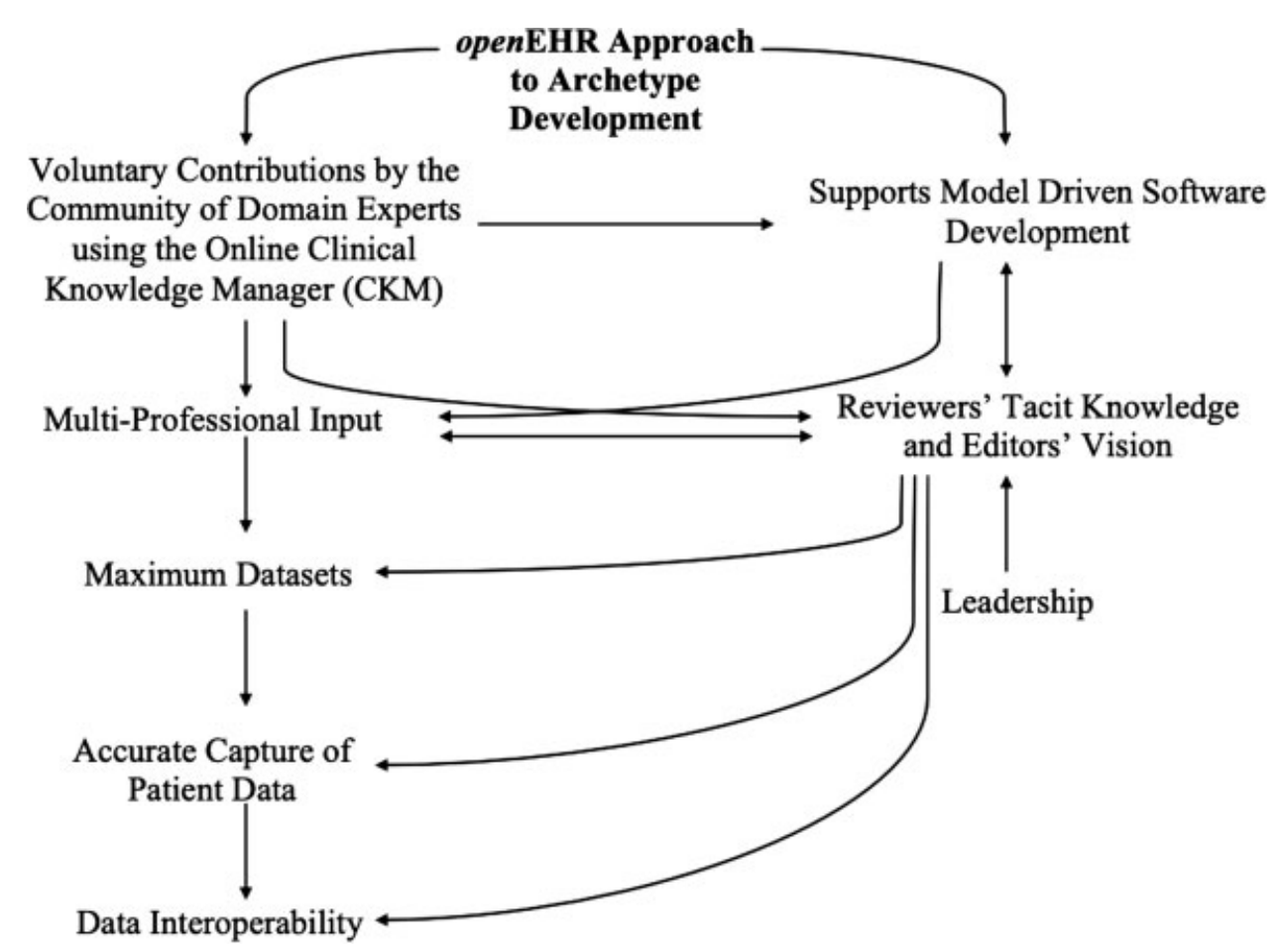

Fig. 3 Aspects of the openEHR approach to archetype development.

Challenges surrounding interoperability, maintainability, and future proofing are among the predominant concerns for the adoption of HIT systems, with particular reference to its future evolution. In this respect, openEHR DCMs are developed with confidence in meeting a multitude of demands between different stakeholders within a health care system. Published DCMs are subject to continuous review and maintenance, and, if need be, further adaptation to meet evershifting health information requirements to address grand health care challenges. ${ }^{2,9,11}$

Having well-defined, shared, and computable models of clinical concepts in the form of DCMS (such as the Tobacco Smoking Summary archetype) is key to the development of HIT systems to support health care systems and professionals to address the rising burden of disease. As all health care professionals have a story to tell, the ability to aggregate their knowledge and experience into a DCM not only ensures the success of the developed HIT system but allows us to gain a better understanding of the health care landscape that surrounds us and therefore a clearer direction as to how we should progress. .10

\section{Data Reuse and Interoperability}

A key element of the openEHR approach is the focus on creating a maximal dataset by incorporating all different data elements from various stakeholders and for different use-cases rather than trying to create a minimum dataset, which has proved to be extremely difficult to achieve using consensus-based development in other similar standards development efforts. ${ }^{32} \mathrm{By}$ using a diverse set of data elements in a DCM to build a particular dataset (using a template), we aim at interoper- ability between different datasets built from common data elements. This approach aims to elicit knowledge from the boundary between unspecifiable and specifiable tacit knowledge as well as implicit knowledge to access the complexity of knowledge in the form of content and process. ${ }^{33}$

The complexity of the health care system, supporting technology, and different viewpoints and requirements of individual users determines interoperability. - Fig. 4 illustrates five tiers of data interoperability ordered in increasing complexity.

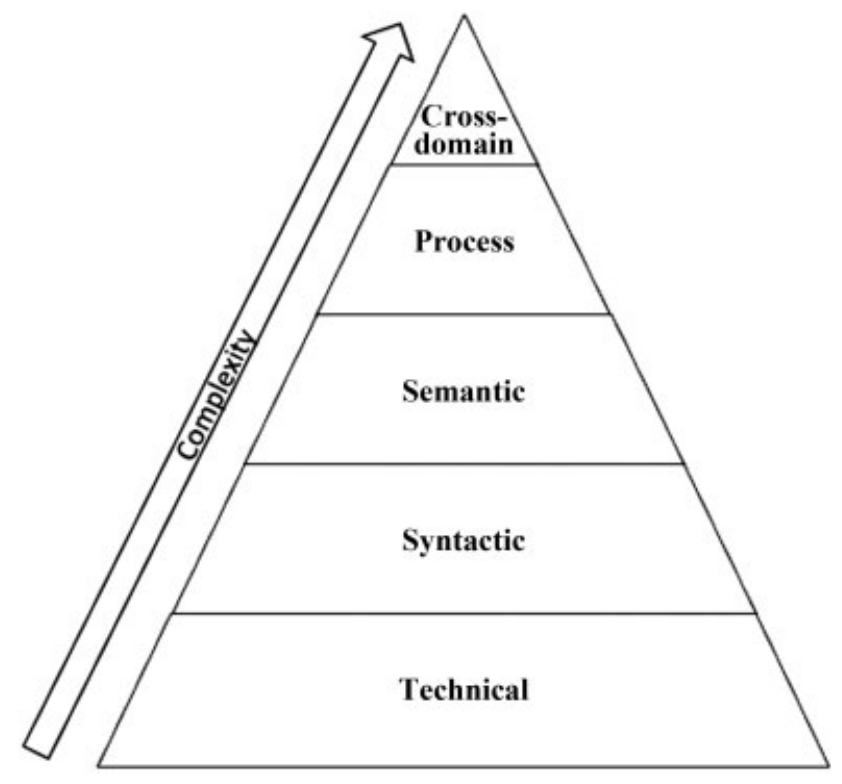

Fig. 4 Tiers of data interoperability. 
Individual stakeholders within the health informatics landscape play a vital role in determining the degree of data interoperability achieved as they influence what is demanded, developed, and adopted. However, due to the differences in cross-sectoral agendas, HIT systems development has a tendency to meet these agendas individually rather than holistically, where the developed systems may not be entirely interoperable. Ideally, HIT solutions should be developed holistically, but this is only possible if stakeholders share a common perspective and understanding toward data interoperability. ${ }^{16,17,34}$

In openEHR, the crowdsourcing of DCM development by a wide range of domain experts not only amalgamates their tacit knowledge to enable knowledge conversion ${ }^{20}$ in the context of DCM development but also brings together the standpoints of many stakeholders, where mutual standards and protocols can be established. The editorial process makes sure that the involved stakeholders align their agendas in the development of HIT solutions, which increases DCM applicability while also addressing barriers of data interoperability. Furthermore, the implications of cross-sectoral agenda alignment within health innovation create the potential to accelerate the rate at which health care systems shift to meet the ever-changing health demands of a population. ${ }^{35,36}$

Currently, there is no matching HL7 FHIR DCM in comparison to the openEHR Tobacco Smoking Archetype. The only relevant artifact is the Smoking Status profile, which is in draft state. ${ }^{37}$ Building on the "observation" resource, it defines a value set comprising eight concepts describing an individual's smoking status from SNOMED CT terminology. ${ }^{38}$

\section{Preventing Reinvention in Health Information Technology}

Successful development of HIT solutions requires a substantial investment of time and resources; for this reason, the approach for progress should be directed toward how we can reuse existing resources rather than reinventing them. ${ }^{39}$ Addressing the rising burden of disease already presents a daunting task for many health systems worldwide. Use of mature DCMs, which reflect the consensus of various stakeholders, helps ensure that new interoperable HIT systems can be built and deployed widely.

The openEHR process for DCM development results in the conversion of tacit knowledge into explicit knowledge. This explicit knowledge then can be shared easily and expanded. In this respect, as health informaticians, we keep to the practices of health innovation by building on existing shared knowledge and ensure the future proofing of developed HIT systems. $^{10,40}$

\section{Study Limitations}

Due to the limited literature available on openEHR, detailed clinical modeling, and data interoperability, there were several unknowns to start with in the beginning of our research. Triangulation of the findings gathered from the observations and interviews compiled multiple perspectives in understanding the same phenomenon and improved the rigor of our research. Considering that the data from the observation and the interviews were aligned with one another and that the findings were consistent with the literature, we can assume that we were able to mitigate this limitation.

\section{Conclusion}

We set out to explore the process that enabled the aggregation of the tacit knowledge of domain experts in an explicit form using the CKM platform and associated assets. We described how crowdsourcing of domain experts contributed to the development of a DCM using the Tobacco Smoking Summary as a case study. We observed experts contributing to the maturation of the existing DCM and also observed the CKM editors' discussions and decision-making regarding changes to the archetype. This was followed by a set of interviews with the reviewers and editors to explore how their tacit knowledge was leveraged for the development and publication of the maximal dataset captured in the Tobacco Smoking Summary DCM.

The openEHR approach to developing an open standard revealed a complex set of conditions for a successful interoperable archetype. These include leadership, maximal dataset, crowdsourced domain expertise and tacit knowledge made explicit, editorial vision, and model-driven software. The adoption of HIT systems relies on flexible interoperability that ideally meets local and global needs simultaneously, hence the development of maximal datasets. Aggregated tacit knowledge that is explicated into a DCM enables the ability to collect accurate data and plan for the future, which, in turn, results in a deeper understanding of the burden of disease related to particular DCMs such as the Tobacco Smoking Summary archetype.

The process based on the CKM platform enables domain experts and stakeholders to be heard and to contribute to mutually designed standards that align local protocols and agendas to international interoperability requirements. This, in turn, provides a platform for sensible innovation and constant improvement in both clinical care and HIT systems. It prevents the reinvention of information technologies by providing enduring open standards that are updated as new knowledge emerges. The robust process captured in the CKM platform enables iterative maturation of archetypes.

On the basis of this research, we recommend that future researchers consider examining (1) the longitudinal process of DCM maturation and its use over years to establish the iterative nature of DCM development and (2) the effect of implementing DCMs in HIT systems to enable enduring interoperability. Our call to action is to embed archetypes in HIT systems to enable local, global, and temporal interoperability as the HIT ecosystem matures.

\section{Clinical Relevance Statement}

This study highlights a health informatics approach to addressing the rising global burden of diseases. The explication of tacit knowledge of domain experts from different domains in the development of clinical models enables the capture of 
necessary data to improve understanding of complex health conditions. Furthermore, these concepts and processes not only enable the accurate capture of patient data, data interoperability, and reusability, but also the future proofing of health systems, thus preventing reinvention.

\section{Multiple Choice Questions}

1. You have signed up in the openEHR CKM as a domain expert for the development of a new DCM. You have a minimum dataset to contribute, but the CKM editors insist on the development of a maximal dataset. Why?

a. Comprehensive data capture is essential for care planning.

b. Accurate data capture is essential for quality clinical care.

c. Diverse data capture is essential for equitable clinical care.

d. Detailed data at any level is essential for quality clinical care.

Correct Answer: The correct answer is option b.

2. As a domain expert in the development of a DCM, you meet and discuss data with diverse stakeholders. Why is it important for stakeholders from different health systems to align their agendas in the interest of interoperability? a. Tact knowledge is shared until a consensus is achieved. b. Mutual standards and protocols can be established using the CKM.

c. When interoperability is achieved data can be confidently reused.

d. CKM editors align stakeholder agendas using the DCM development process.

Correct Answer: The correct answer is option c.

\section{Protection of Human and Animal Subjects}

This study has satisfied the ethical considerations of research required by the University of Auckland Human Participation Ethics Committee and was approved on August 19, 2016, for 3 years (reference number: 017360).

\section{Conflict of Interest}

None declared.

\section{Acknowledgments}

We would like to thank the editors of the CKM involved in this research for their generous invitation to the first author to observe the reviews and editorial discussions. We would also like to thank the domain experts who participated in the reviews and interviews for their time and thoughtful insights.

\section{References}

1 Greenhalgh EM, Stillman S, Ford C. Factors that predict success or failure in quit attempts. In: Scollo MM, Winstanley MH, eds. Tobacco in Australia: Facts and Issues. Melbourne: Cancer Council Victoria; 2016
2 Habib JL. EHRs, meaningful use, and a model EMR. Drug Benefit Trends 2010;22(04):99-101

3 Heeks R. Health information systems: failure, success and improvisation. Int J Med Inform 2006;75(02):125-137

4 Kruse CS, Stein A, Thomas H, Kaur H. The use of electronic health records to support population health: a systematic review of the literature. J Med Syst 2018;42(11):214

5 Kalra D, Beale T, Heard S. The openEHR Foundation. Stud Health Technol Inform 2005;115:153-173

6 International Telecommunications Union. Definition of "Open Standards."2018. Available at: https://www.itu.int/en/ITU-T/ipr/Pages/ open.aspx. Accessed December 10, 2018

7 Martínez-Costa C, Menárguez-Tortosa M, Fernández-Breis JT. An approach for the semantic interoperability of ISO EN 13606 and OpenEHR archetypes. J Biomed Inform 2010;43(05):736-746

8 Goossen W, Goossen-Baremans A, van der Zel M. Detailed clinical models: a review. Healthc Inform Res 2010;16(04):201-214

9 Kropf S, Chalopin C, Lindner D, Denecke K. Domain modeling and application development of an archetype- and XML-based EHRS. Practical experiences and lessons learnt. Appl Clin Inform 2017;8 (02):660-679

10 Brabham DC. Crowdsourcing as a model for problem solving: an introduction and cases. Convergence 2008;14(01):75-90

11 Pahl C, Zare M, Nilashi M, et al. Role of OpenEHR as an open source solution for the regional modelling of patient data in obstetrics. J Biomed Inform 2015;55:174-187

12 Saleem MQ Jaafar J, Hassan MF. Model Driven Software Development: An Overview. Presented in IEEE International Conference on Computer and Information Sciences (ICCOINS), Kuala Lumpur, Malaysia, June 3-5, 2014

13 Atkinson C, Kuhne T. Model-driven development: a metamodeling foundation. IEEE Softw 2003;20(05):36-41

14 Bird L, Goodchild A, Tun Z. Experiences with a two-level modelling approach to electronic health records. J Res Pract Inf Technol 2003;35(02):121

15 de Moraes JLC, de Souza WL, Pires LF, Cavalini LT, Do Prado AF. Using the dual-level modeling approach to developing applications in the pervasive healthcare environment. J Mobile Multimedia 2013;9(1\&2):111-127

16 Christensen B, Ellingsen G. Evaluating Model-Driven Development for large-scale EHRs through the openEHR approach. Int J Med Inform 2016;89:43-54

17 Ocean Health Systems. Clinical Knowledge Manager. Available at: http://oceanhealthsystems.com/products/clinical-knowledgemanager. Accessed December 10, 2018

18 Garde S, Chen R, Leslie H, Beale T, McNicoll I, Heard S. Archetypebased knowledge management for semantic interoperability of electronic health records. Stud Health Technol Inform 2009; 150:1007-10011

19 Selic B. The pragmatics of model-driven development. IEEE Softw 2003;20(05):19-25

20 Nonaka I, Toyama R, Konno N. SECI, Ba and leadership: a unified model of dynamic knowledge creation. Long Range Plann 2000;33 (01):5-34

21 Muñoz CA, Mosey S, Binks M. The tacit mystery: reconciling different approaches to tacit knowledge. Knowl Manag Res Pract 2015;13(03):289-298

22 Nonaka I, von Krogh G. Tacit knowledge and knowledge conversion: controversy and advancement in organisational knowledge creation theory. Organ Sci 2009;20(03):635-652

23 Atalag K, Yang HY, Warren J. On the Maintainability of openEHR Based Health Information Systems-an Evaluation Study in Endoscopy. Proceedings of the Health Informatics Society of Australia. 18th Annual Health Informatics Conference: Informing the Business of Healthcare, August 24-26, 2010, Melbourne Convention and Exhibition Centre

24 HL7. FHIR Release 3 (STU). 2017. Available at: http://www.hl7. org/fhir/. Accessed December 10, 2018 
25 Lee J, Hulse NC, Wood GM, Oniki TA, Huff SM. Profiling Fast Healthcare Interoperability Resources (FHIR) of family health history based on the clinical element models. AMIA Annu Symp Proc 2016:753-762

26 Hsieh H-F, Shannon SE. Three approaches to qualitative content analysis. Qual Health Res 2005;15(09):1277-1288

27 Paltridge B. Discourse Analysis: An Introduction (2nd ed.). London; New York: Bloomsbury Publishing; 2012

28 Thomas DR. A general inductive approach for analysing qualitative evaluation data. Am J Eval 2006;27(02):237-246

29 Braun V, Clarke V. What can "thematic analysis" offer health and wellbeing researchers? Int J Qual Stud Health Well-being 2014; 9:26152

30 openEHR. Tobacco smoking summary (latest revision/latest published). 2016]. Available at: https://openehr.org/ckm/\#showArchetype_1013.1.2466_TABBED. Accessed December 10, 2018

31 Morse JM. Critical analysis of strategies for determining rigor in qualitative inquiry. Qual Health Res 2015;25(09):1212-1222

32 Leslie H. Anatomy of an archetype. Archetypical 2011 [cited 2018 10 December 2018]; Available at: https://omowizard.wordpress. com/2011/02/18/anatomy-of-an-archetype/

33 Snowden D. Complex acts of knowing: paradox and descriptive self-awareness. Bull Am Soc Inf Sci Technol 2003;29(04):23-28
34 Miller P. Interoperability. What Is It and Why Should I Want It? Ariadne Issue 24. 2000. Available at: http://www.ariadne.ac.uk/ issue24/interoperability/. Accessed December 10, 2018

35 Marcos M, Martínez-Salvador B. Towards the interoperability of computerised guidelines and electronic health records: an experiment with openEHR archetypes and a chronic heart failure guideline. In: International Workshop on Knowledge Representation for Health Care 2010 Aug 17 (pp. 101-113). Heidelberg; Berlin: Springer

36 Millard DE, Davis HC. Navigating spaces: the semantics of cross domain interoperability. In: International Workshop on Structural Computing. Heidelberg: Springer; 2010

37 HL7. FHIR US Core Implementation Guide (Release 1.1.0 Ballot) (a). 2017. Available at: http://hl7.org/fhir/us/core/2018Jan/StructureDefinition-us-core-smokingstatus.html. Accessed December 10, 2018

38 HL7. FHIR US Core Implementation Guide (Release 1.1.0 Ballot) (b). 2017. Available at: http://hl7.org/fhir/us/core/2018Jan/ValueSet-us-core-observation-ccdasmokingstatus.html. Accessed December 10, 2018

39 Deitering A-M, Bridgewater R. Stop reinventing the wheel: using wikis for professional knowledge sharing. J Web Librariansh 2007;1(01):27-44

40 Mooney BL, Boyle AM. 10 steps to successful EHR implementation. Med Econ 2011;88(09):S4-S6 